to large quantities of sea water, to which ammonium chloride had been added beforehand. The thorium content was found to be certainly less than $10^{-6} \mathrm{gm}$. thorium per litre and probably less than half that amount. If we assume the removal of thorium from sea water through natural precipitation with iron to be as efficient as the removal of ionium, taking the thorium-uranium ratio in the denuded rocks to be $2: 1$, we should expect the thorium content in sea water (judging from its uranium content) to be reduced from, say, $4 \times 10^{-6} \mathrm{gm}$. thorium per litre to one tenth that amount, that is, to $4 \times 10^{-7} \mathrm{gm}$. thorium per litre, or very probably still further.

A detailed report on our measurements will appear in Meddelanden från Oceanografiska Institutet $i$ Göteborg.

Since writing this account of our work, our attention has been directed to a paper by R. D. Evans, Arthur F. Kip and E. G. Moberg ${ }^{3}$; working by different methods and using much smaller quantities of sea water, these investigators have found values for the radium content not much different from those we have found with water of quite different origin.

Bornö Station, Sweden.

${ }^{1}$ Medd. Ocean. Inst., No. 11 (1937).

${ }^{2}$ Wiener Akad. d. Wissenschaften, July 1, 1937.

${ }^{3}$ Amer. J. Science, Oct. 1938.

\section{Physical Evidence for the Division of Heavy Nuclei} under Neutron Bombardment

From chemical evidence, Hahn and Strassmann ${ }^{1}$ conclude that radioactive barium nuclei (atomic number $Z=56)$ are produced when uranium $(Z=92)$ is bombarded by neutrons. It has been pointed out ${ }^{2}$ that this might be explained as a result of a 'fission' of the uranium nucleus, similar to the division of a droplet into two. The energy liberated in such processes was estimated to be about $200 \mathrm{Mev}$., both from mass defect considerations and from the repulsion of the two nuclei resulting from the 'fission' process.

If this picture is correct, one would expect fastmoving nuclei, of atomic number about 40-50 and atomic weight 100-150, and up to $100 \mathrm{Mev}$. energy, to emerge from a layer of uranium bombarded with neutrons. In spite of their high energy, these nuclei should have a range, in air, of a few millimetres only, on account of their high effective charge (estimated to be about 20), which implies very dense iodization. Each such particle should produce a total of about three million ion pairs.

By means of a uranium-lined ionization chamber, connected to a linear amplifier, I have succeeded in demonstrating the occurrence of such bursts of ionization. The amplifier was connected to a thyratron which was biased so as to count only pulses corresponding to at least $5 \times 10^{5}$ ion pairs. About fifteen particles a minute were recorded when $300 \mathrm{mgm}$. of radium, mixed with beryllium, was placed one centimetre from the uranium lining. No pulses at all were recorded during repeated check runs of several hours total duration when either the neutron source or the uranium lining was removed. With the neutron source at a distance of four centimetres from the uranium lining, surrounding the source with paraffin wax enhanced the effect by a factor of two.
It was checked that the number of pulses depended linearly on the strength of the neutron source; this was done in order to exclude the possibility that the pulses are produced by accidental summation of smaller pulses. When the amplifier was connected to an oscillograph, the large pulses could be seen very distinctly on the background of much smaller pulses due to the alpha particles of the uranium.

By varying the bias of the thyratron, the maximum size of pulses was found to correspond to at least two million ion pairs, or an energy loss of $70 \mathrm{Mev}$. of the particle within the chamber. Since the longest path of a particle in the chamber was three centimetres and the chamber was filled with hydrogen at atmospheric pressure, the particles must ionize so heavily, in spite of their energy of at least $70 \mathrm{Mev}$., that they can make two million ion pairs on a path equivalent to $0.8 \mathrm{~cm}$. of air or less. From this it can be estimated that the ionizing particles must have an atomic weight of at least about seventy, assuming a reasonable connexion between atomic weight and effective charge. This seems to be conclusive physical evidence for the breaking up of uranium nuclei into parts of comparable size, as indicated by the experiments of Hahn and Strassmann.

Experiments with thorium instead of uranium gave quite similar results, except that surrounding the neutron source with paraffin did not enhance, but slightly diminished, the effect. This gives evidence in favour of the suggestion ${ }^{2}$ that also in the case of thorium, some, if not all, of the activities produced by neutron bombardment ${ }^{3}$ should be ascribed to light elements. It should be remembered that no enhancement by paraffin has been found for the activities produced in thorium ${ }^{3}$ (except for one which is isotopic with thorium and is almost certainly produced by simple capture of the neutron).

Prof. Meitner has suggested another interesting experiment. If a metal plate is placed close to a uranium layer bombarded with neutrons, one would expect an active deposit of the light atoms emitted in the 'fission' of the uranium to form on the plate. We hope to carry out such experiments, using the powerful source of neutrons which our high-tension apparatus will soon be able to provide.

Institute of Theoretical Physics,

O. R. Frisch.

$$
\begin{gathered}
\text { University, } \\
\text { Copenhagen. } \\
\text { Jan. } 16 .
\end{gathered}
$$

${ }^{\mathrm{t}}$ Hahn, O., and Strassmann, F., Naturwis8., 27, 11 (1939).

- Meitner, L., and Frisch, O. R., NATURE [143, 239 (1939)].

${ }^{3}$ See Meitner, L., Strassmann, F., and Hahn, O., Z. Phys., 109, 538 (1938).

\section{The Fundamental Length Introduced by the Theory of the Mesotron (Meson)*}

Ir is well known that the vector theory of the meson $^{1}$ contains a fundamental length in the interaction of mesons with protons and neutrons determined by the fact that the mass of the meson appears * The name 'mesotron' has been suggested by Anderson and Nedder-
meyer (NATURE, 142, 874; 1938) for the new particle found in cosmic
radiation with a mass intermediate between that of the electron and
proton. It is felt that the 'tr' in this word is redundant, since it does
not belong to the Greek root 'meso' for middle ; the "tr' in neutron
and electron belong, of course, to the roots "neutr" and "electra".
In these circumstances, it seems better to follow the suggestion of
Bohr and to use electron to denote particles of electronic mass inde-
pendently of their charge, and negaton and positon to differentiate
between the sign of the charge. It would therefore be more logical and
also shorter to call the new particle a meson instead of a mesotron. 